\title{
The number of powers of 2 in a representation of large even integers by sums of such powers and of two primes (II)
}

by

Hongze Li (Jinan)

1. Main results. The Goldbach conjecture is that every integer not less than 6 is a sum of two odd primes. The conjecture still remains open. Let $E(x)$ denote the number of positive even integers not exceeding $x$ which cannot be written as a sum of two prime numbers. In 1975 Montgomery and Vaughan [15] proved that

$$
E(x) \ll x^{1-\theta}
$$

for some small computable constant $\theta>0$. For the number $\theta$, see [1]-[3]. In [5] the author proved that $E(x) \ll x^{0.921}$, recently in [6] the author improved the result to $E(x) \ll x^{0.914}$.

In 1951 and 1953, Linnik [8, 9] established the following "almost Goldbach" result.

Every large positive even integer $N$ is a sum of two primes $p_{1}, p_{2}$ and a bounded number of powers of 2, i.e.

$$
N=p_{1}+p_{2}+2^{\nu_{1}}+\ldots+2^{\nu_{k}} .
$$

Let $r_{k}^{\prime \prime}(N)$ denote the number of $N$ in the form (1.1). In [10] Liu, Liu and Wang proved that for any $k \geq 54000$, there exists $N_{k}>0$ depending on $k$ only such that if $N \geq N_{k}$ is an even integer then

$$
r_{k}^{\prime \prime}(N) \gg N(\log N)^{k-2} .
$$

Recently in [7] the author improved the constant to $k \geq 25000$. In this paper we prove the following result.

THEOREM 1 . For any integer $k \geq 1906$, there exists $N_{k}>0$ depending on $k$ only such that if $N \geq N_{k}$ is an even integer then

$$
r_{k}^{\prime \prime}(N) \gg N(\log N)^{k-2} \text {. }
$$

2000 Mathematics Subject Classification: 11P32, 11P55.

Project supported by The National Natural Science Foundation of China. 
Let $r_{k}^{\prime}(n)$ denote the number of representations of an odd integer $n$ in the form

$$
n=p+2^{\nu_{1}}+\ldots+2^{\nu_{k}} .
$$

The second purpose of this paper is to establish the following result.

TheOREM 2. For any $\varepsilon>0$, there exists a constant $k_{0}$ depending on $\varepsilon$ only such that if $k \geq k_{0}, N \geq N_{k}$ then

$$
\sum_{2 \nmid n \leq N}\left(r_{k}^{\prime}(n)-2\left(\log _{2} N\right)^{k}(\log N)^{-1}\right)^{2} \leq \varepsilon 2 N\left(\log _{2} N\right)^{2 k}(\log N)^{-2} .
$$

In particular, for $\varepsilon=0.9986, k_{0}=953$ is permissible.

In what follows, $L(s, \chi)$ denotes the Dirichlet $L$-function. $\varepsilon$ denotes a positive constant which is arbitrarily small but not necessarily the same at each occurrence. $A$ will be sufficiently large, $A<P$.

2. Some lemmas. Let $N$ be a large integer,

$$
\theta:=\frac{1}{13}, \quad P:=N^{\theta}, \quad T:=P^{2.01}, \quad Q:=P^{-1} N(\log N)^{-3}, \quad D=P^{1+\varepsilon} .
$$

Let $A<q \leq P$, and let $\chi_{q}$ be a non-principal character $\bmod q$. Write $\alpha=1-\lambda / \log D$. Assume that

$$
\alpha \leq \sigma \leq 1, \quad|t| \leq D / q .
$$

Lemma 1. Suppose $P$ is sufficiently large. Then no function $L(s, \chi)$ with $\chi$ primitive modulo $q \leq P$, except for a possible exceptional one only, has a zero in the region

$$
\sigma \geq 1-\frac{0.239}{\log P}, \quad q(|t|+1) \leq P^{1+\varepsilon} .
$$

If the exceptional function exists, denoted by $L(s, \tilde{\chi})$, then $\tilde{\chi}$ must be a real primitive character modulo $\widetilde{q}, \widetilde{q} \leq P$, and $L(s, \widetilde{\chi})$ has a real simple zero $\widetilde{\beta}$ which satisfies

$$
1-\frac{0.239}{\log P} \leq \widetilde{\beta} \leq 1-\frac{c}{\widetilde{q}^{10^{-8}}}
$$

This is Lemma 2.3 of [5].

For $q_{1}, q_{2} \leq P$, we now consider the zeros of $L\left(s, \chi_{q_{1}}\right)$ and $L\left(s, \chi_{q_{2}}\right)$ for non-principal characters $\chi_{q_{1}}$ and $\chi_{q_{2}}$. If $\varrho_{1}=\beta_{1}+i \gamma_{1}=1-\lambda_{1} / \log P+i \gamma_{1}$ is a zero of $L\left(s, \chi_{q_{1}}\right)$ satisfying $q_{1}\left(\left|\gamma_{1}\right|+1\right) \leq P^{1+\varepsilon}$, and $\varrho_{2}=\beta_{2}+i \gamma_{2}=$ $1-\lambda_{2} / \log P+i \gamma_{2}$ is a zero of $L\left(s, \chi_{q_{2}}\right)$ satisfying $q_{2}\left(\left|\gamma_{2}\right|+1\right) \leq P^{1+\varepsilon}$, then we have the lower bounds for $\lambda_{2}$ given in Table 1 . 
Table 1. The lower bound for $\lambda_{2}$

\begin{tabular}{c|c}
\hline$\lambda_{1}$ & $\lambda_{2}$ \\
\hline 0.24 & 0.444 \\
0.26 & 0.418 \\
0.28 & 0.393 \\
0.30 & 0.37 \\
0.32 & 0.349 \\
0.334 & 0.334 \\
\hline
\end{tabular}

If $\left[q_{1}, q_{2}\right] \leq P^{\varepsilon}\left(q_{1}, q_{2}\right)$, then we have the following lower bounds for $\lambda_{2}$ :

Table 2. The lower bound for $\lambda_{2}$

\begin{tabular}{c|c|c|c}
\hline$\lambda_{1}$ & $\lambda_{2}$ & $\lambda_{1}$ & $\lambda_{2}$ \\
\hline 0.22 & 1.189 & 0.38 & 0.745 \\
0.24 & 1.116 & 0.40 & 0.706 \\
0.26 & 1.050 & 0.42 & 0.669 \\
0.28 & 0.989 & 0.44 & 0.634 \\
0.30 & 0.933 & 0.46 & 0.601 \\
0.32 & 0.881 & 0.48 & 0.570 \\
0.34 & 0.832 & 0.50 & 0.541 \\
0.36 & 0.787 & 0.517 & 0.517 \\
\hline
\end{tabular}

The entry $0.40,0.706$ indicates that $\lambda_{2} \geq 0.706$ whenever $\lambda_{1} \leq 0.40$ (see [5]).

Let $S_{j q}=\left\{\chi_{q}: L\left(s, \chi_{q}\right)\right.$ has only $j$ zeros in the region (2.2)\}. Suppose $A<q_{0} \leq P$, define

$$
\begin{aligned}
& N_{1}^{*}(\alpha, P)=N_{1}^{*}(\lambda, P)=\sum_{\substack{A<q \leq P \\
\left[q, q_{0}\right] \leq D^{\varepsilon}\left(q, q_{0}\right)}} \sum_{j \geq 1} \sum_{\chi \in S_{j q}}^{*} j, \\
& N^{*}(\alpha, P)=N^{*}(\lambda, P)=\sum_{A<q \leq P} \sum_{j \geq 1} \sum_{\chi \in S_{j q}}^{*} j
\end{aligned}
$$

where $\sum^{*}$ indicates that the sum is over primitive characters.

Lemma 2. Suppose $A<q_{0} \leq P, 0<\lambda \leq \varepsilon \log D$. Then

$$
N_{1}^{*}(\alpha, P)=N_{1}^{*}(\lambda, P) \leq \begin{cases}4.356 C_{1}(\lambda) e^{4.064 \lambda}, & 0.517<\lambda \leq 0.575, \\ 8.46 C_{2}(\lambda) e^{4.12 \lambda}, & 0.575<\lambda \leq 0.618, \\ 14.3 C_{3}(\lambda) e^{4.5 \lambda}, & 0.618<\lambda \leq 1, \\ 104.1 C_{4}(\lambda) e^{3.42 \lambda}, & 1<\lambda \leq 5, \\ 268.6 e^{2.16 \lambda}, & 5<\lambda \leq \varepsilon \log D .\end{cases}
$$


where

$$
N^{*}(\alpha, P)=N^{*}(\lambda, P) \leq \begin{cases}3.632 C_{5}(\lambda) e^{5.2 \lambda}, & 0.334<\lambda \leq 0.517 \\ 4.338 C_{6}(\lambda) e^{4.82 \lambda}, & 0.517<\lambda \leq 0.575, \\ 10.42 C_{7}(\lambda) e^{4.5 \lambda}, & 0.575<\lambda \leq 0.618 \\ 14.91 C_{8}(\lambda) e^{5.2 \lambda}, & 0.618<\lambda \leq 1, \\ 104.8 C_{9}(\lambda) e^{4.16 \lambda}, & 1<\lambda \leq 5, \\ 279.7 e^{2.9 \lambda}, & 5<\lambda \leq \varepsilon \log D,\end{cases}
$$

$$
\begin{aligned}
& C_{1}(\lambda)=\lambda^{-1}\left(1-e^{-4.064 \lambda} \frac{e^{2.808 \lambda}-e^{1.76 \lambda}}{1.048 \lambda}\right) \\
& C_{2}(\lambda)=\lambda^{-1}\left(1-e^{-4.12 \lambda} \frac{e^{2.855 \lambda}-e^{1.78 \lambda}}{1.075 \lambda}\right) \\
& C_{3}(\lambda)=\lambda^{-1}\left(1-e^{-4.5 \lambda} \frac{e^{3.198 \lambda}-e^{2.013 \lambda}}{1.185 \lambda}\right) \\
& C_{4}(\lambda)=\lambda^{-1}\left(1-e^{-3.42 \lambda} \frac{e^{2.358 \lambda}-e^{1.64 \lambda}}{0.718 \lambda}\right) \\
& C_{5}(\lambda)=\lambda^{-1}\left(1-e^{-5.2 \lambda} \frac{e^{3.866 \lambda}-e^{2.668 \lambda}}{1.198 \lambda}\right) \\
& C_{6}(\lambda)=\lambda^{-1}\left(1-e^{-4.82 \lambda} \frac{e^{3.565 \lambda}-e^{2.51 \lambda}}{1.055 \lambda}\right) \\
& C_{7}(\lambda)=\lambda^{-1}\left(1-e^{-4.5 \lambda} \frac{e^{3.32 \lambda}-e^{2.36 \lambda}}{0.96 \lambda}\right) \\
& C_{8}(\lambda)=\lambda^{-1}\left(1-e^{-5.2 \lambda} \frac{e^{3.928 \lambda}-e^{2.7312 \lambda}}{1.1968 \lambda}\right) \\
& C_{9}(\lambda)=\lambda^{-1}\left(1-e^{-4.16 \lambda} \frac{e^{3.104 \lambda}-e^{2.38 \lambda}}{0.724 \lambda}\right)
\end{aligned}
$$

This is Lemma 6 of [6].

3. The major arcs. By Dirichlet's lemma on rational approximations, each $\alpha \in\left[Q^{-1}, 1+Q^{-1}\right]$ may be written in the form

$$
\alpha=a / q+\lambda, \quad|\lambda| \leq(q Q)^{-1},
$$

for some positive integers $a, q$ with $1 \leq a \leq q,(a, q)=1$ and $q \leq Q$. We denote by $I(a, q)$ the set of $\alpha$ satisfying $(3.1)$, and put

$$
E_{1}=\bigcup_{q \leq P} \bigcup_{\substack{a=1 \\(a, q)=1}}^{q} I(a, q), \quad E_{2}=\left[Q^{-1}, 1+Q^{-1}\right]-E_{1} .
$$

When $q \leq P$ we call $I(a, q)$ a major arc. By (2.1), all major arcs are mutually disjoint. Let $e(\alpha)=\exp (i 2 \pi \alpha)$ and $S(\alpha)=\sum_{N^{1-\varepsilon}<p \leq N} e(p \alpha)$. 
Let $\sigma(n)$ denote the singular series in the Goldbach problem, i.e.

$$
\sigma(n):=\prod_{p \mid n}\left(1+(p-1)^{-1}\right) \prod_{p \nmid n}\left(1-(p-1)^{-2}\right) \gg 1
$$

for even $n$. Let

$$
J(n):=\sum_{\substack{1<n_{1}, n_{2} \leq N \\ n_{1}-n_{2}=n}}\left(\log n_{1} \log n_{2}\right)^{-1} .
$$

Theorem 3. Let $n$ with $|n| \leq N^{2}$ be a non-zero integer, and let $P, Q$ satisfy (2.1). Then for even $n$ we have

where

$$
\int_{E_{1}}|S(\alpha)|^{2} e(n \alpha) d \alpha=\sigma(n) J(n)+R,
$$

$$
|R| \leq \sigma(n) N(\log N)^{-2}\left\{0.11943387+O\left(\widetilde{q} \phi((n, \widetilde{q})) / \phi^{2}(\widetilde{q})\right)\right\},
$$

the $O$ term occurring only when there exists $\widetilde{\beta}$ in Lemma 1.

Let

$$
r_{0}(n)=\int_{E_{1}}|S(\alpha)|^{2} e(n \alpha) d \alpha, \quad S(\lambda, \chi)=\sum_{N^{1-\varepsilon}<p \leq N} \chi(p) e(p \lambda),
$$

$T(\lambda)=\sum_{N^{1-\varepsilon}<m \leq N} e(m \lambda) / \log m, \quad \widetilde{T}(\lambda)=-\sum_{N^{1-\varepsilon}<m \leq N} m^{\widetilde{\beta}-1} e(m \lambda) / \log m$,

and

$$
\begin{cases}S\left(\lambda, \chi_{q}^{0}\right)=T(\lambda)+W\left(\lambda, \chi_{q}^{0}\right) & \\ S\left(\lambda, \chi_{q}^{0} \widetilde{\chi}\right)=\widetilde{T}(\lambda)+W\left(\lambda, \chi_{q}^{0} \widetilde{\chi}\right) & \text { if } \widetilde{q} \mid q \\ S\left(\lambda, \chi_{q}\right)=W\left(\lambda, \chi_{q}\right) & \text { otherwise }\end{cases}
$$

$G(m, \chi)=\sum_{h=1}^{q} \chi(h) e\left(\frac{m h}{q}\right), \quad \tau(\chi)=G(1, \chi), \quad C_{q}(m)=\sum_{\substack{h \leq q \\(h, q)=1}} e\left(\frac{m h}{q}\right)$.

As in (4.7) of [11], we have

$$
r_{0}(n)=\sum_{j=1}^{9} r_{j}(n)+O\left(P^{2}(\log N)^{3}\right) .
$$

For the definitions of $r_{j}(n)$, see [11].

LEMMA 3.

$$
\begin{gathered}
r_{1}(n)=\sigma(n) J(n)+O\left(N(\log N)^{-3}\right), \\
r_{2}(n), r_{3}(n) \ll \frac{\widetilde{q}}{\phi^{2}(\widetilde{q})} \cdot \frac{N}{(\log N)^{2}} \sigma(n), \quad r_{4}(n) \ll \frac{\widetilde{q} \phi((n, \widetilde{q}))}{\phi^{2}(\widetilde{q})} \cdot \frac{N}{(\log N)^{2}} \sigma(n), \\
r_{5}(n), r_{6}(n) \ll \frac{N}{(\log N)^{6}} \sigma(n) .
\end{gathered}
$$


Proof. Apply Lemmas 14 and 16 of [11] (note that $W \ll 1$ ).

Lemma 4. Let $\chi_{i}$ be primitive characters $\left(\bmod r_{i}\right), i=1,2, r=\left[r_{1}, r_{2}\right]$. Then for $m \neq 0$,

$$
\begin{gathered}
\sum_{\substack{q \leq P \\
r \mid q}} \phi(q)^{-2}\left|G\left(m, \bar{\chi}_{1} \bar{\chi}_{2} \chi_{0}\right) \tau\left(\chi_{1} \chi_{0}\right) \tau\left(\chi_{2} \chi_{0}\right)\right| \leq 2.140782 \sigma(m), \\
\sum_{\substack{q \leq P \\
r \mid q}} \phi(q)^{-2}\left|G\left(m, \bar{\chi}_{1} \bar{\chi}_{2} \chi_{0}\right) \tau\left(\chi_{1} \chi_{0}\right) \tau\left(\chi_{2} \chi_{0}\right)\right| \ll\left(r_{1}, r_{2}\right) r^{-1} \sigma(m) \log P .
\end{gathered}
$$

Proof. This lemma is similar to Lemma 5.5 of [15], but our proof is similar to that of Lemma 5.2 of [14]. Define

$$
\begin{array}{r}
Z\left(q, \chi_{1}, \chi_{2}\right):=\sum_{\substack{h=1 \\
(h, q)=1}}^{q} e\left(\frac{h m}{q}\right) \prod_{j=1}^{2} G\left(h, \chi_{j} \chi_{0}\right) \\
=\left|G\left(m, \bar{\chi}_{1} \bar{\chi}_{2} \chi_{0}\right) \tau\left(\chi_{1} \chi_{0}\right) \tau\left(\chi_{2} \chi_{0}\right)\right|, \\
A(q):=\phi(q)^{-2} \sum_{\substack{h=1 \\
(h, q)=1}}^{q} e\left(\frac{h m}{q}\right) \prod_{j=1}^{2} G\left(h, \chi_{0}\right) .
\end{array}
$$

By Lemma 4.1 of [13], we know $A(q)$ is multiplicative. For any prime $p$, let

$$
s(p):=1+A(p) .
$$

Since $A(p)=1 /(p-1)$ if $p \mid m$ and $A(p)=-1 /(p-1)^{2}$ if $p \nmid m$, similarly to Lemma 4.6 of [13] and Lemma 5.2 of [14], the first inequality holds. By the proof of Lemma 5.5 of [15], the second inequality holds.

Let

$$
\begin{gathered}
W\left(\chi_{d}\right)=\left(\int_{-1 /(d Q)}^{1 /(d Q)}\left|W\left(\lambda, \chi_{d}\right)\right|^{2} d \lambda\right)^{1 / 2}, \\
W(P)=\sum_{d \leq P} \sum_{\chi_{d}}^{*} W\left(\chi_{d}\right),
\end{gathered}
$$

where $*$ indicates that the sum is over for primitive characters $\chi_{d}$; and

$$
\begin{gathered}
W(P, \widetilde{q})=\sum_{\substack{d \leq P \\
[d, \widetilde{q}] \leq D^{\varepsilon}(d, \widetilde{q})}} \sum_{\chi_{d}}^{*} W\left(\chi_{d}\right), \\
W^{\prime}(P)=\max \sum_{\substack{d \leq P \\
\left[d_{1}, d\right] \leq D^{\varepsilon}\left(d_{1}, d\right)}} \sum_{\chi_{d}}^{*} W\left(\chi_{d}\right) .
\end{gathered}
$$

Here the max is over $A<d_{1} \leq P$. 
Similarly to Section III of [2] we have

$$
\begin{aligned}
W\left(\chi_{d}\right) \leq & \left(1+2 \cdot 10^{-5}\right)\left(N^{1 / 2} / \log N\right) \sum_{\substack{\beta \geq 1 / 4 \\
\left|\gamma_{\chi_{d}}\right| \leq P^{1+\varepsilon} d^{-1}}}^{\prime} N^{(1-\varepsilon)(\beta-1)} \\
& +O\left(N^{1 / 2-\varepsilon} \sum_{\substack{\beta \geq 1 / 4 \\
\left|\gamma_{\chi_{d}}\right| \leq P^{1.01} d^{-1}}}^{\prime \beta-1}\right) \\
& +O\left(P^{1 / 2-0.01 \theta} \sum_{\substack{\beta \geq 1 / 4 \\
\left|\gamma_{\chi_{d}}\right| \leq P^{2.01}}}^{\prime} N^{\beta-1}\right)+O\left(N^{1 / 2-1.01 \theta+\varepsilon} d^{-1}\right),
\end{aligned}
$$

where $\sum^{\prime}$ indicates that the sum does not contain the exceptional zero $\widetilde{\beta}$.

By the same methods as used in [1] we have

$$
\begin{gathered}
\sum_{\substack{d \leq P \\
\left[d_{1}, d\right] \leq D^{\varepsilon}\left(d_{1}, d\right)}} \sum_{\chi_{d}}^{*} \sum_{\substack{\beta \geq 1 / 4 \\
\gamma_{\chi_{d}} \mid \leq P^{2.01}}}^{\prime} N^{\beta-1} \ll N^{0.7 \varepsilon}, \\
\sum_{d \leq P} \sum_{\chi_{d}}^{*} \sum_{\substack{\beta \geq 1 / 4 \\
\left|\gamma_{\chi_{d}}\right| \leq P^{2.01}}}^{\prime} N^{\beta-1} \ll N^{0.7 \varepsilon} .
\end{gathered}
$$

Let

$$
\begin{aligned}
I_{1} & =\sum_{\substack{d \leq P \\
\left[d_{1}, d\right] \leq D^{\varepsilon}\left(d_{1}, d\right)}} \sum_{\chi_{d}}^{*} \sum_{\substack{\beta \geq 1 / 4 \\
\left|\gamma_{\chi_{d}}\right| \leq P^{1+\varepsilon} d^{-1}}}^{\prime} N^{(1-\varepsilon)(\beta-1)}, \\
I_{2} & =\sum_{d \leq P} \sum_{\chi_{d}}^{*} \sum_{\substack{\beta \geq 1 / 4 \\
\left|\gamma_{\chi_{d}}\right| \leq P^{1+\varepsilon} d^{-1}}}^{\prime} N^{(1-\varepsilon)(\beta-1)} .
\end{aligned}
$$

Suppose $\varrho_{\chi_{d}}=\beta_{\chi_{d}}+i \gamma_{\chi_{d}},\left|\gamma_{\chi_{d}}\right| \leq P^{1+\varepsilon} d^{-1}$, is a zero of $L\left(s, \chi_{d}\right)$. Let $\mathcal{L}=(1+\varepsilon) \log P$.

1) If $1-0.24 / \mathcal{L} \leq \beta_{\chi_{d}} \leq 1-0.239 / \mathcal{L}$, then by Lemma 1 and Tables 1 and 2 we have

$$
\begin{aligned}
& I_{1} \leq 2 e^{-0.239 /(\theta+\varepsilon)}+\frac{1}{\theta+\varepsilon} \int_{1.116}^{\infty} e^{-(1-\varepsilon) t /(\theta+\varepsilon)} N_{1}^{*}(t, P) d t \leq 0.091628, \\
& I_{2} \leq 2 e^{-0.239 /(\theta+\varepsilon)}+\frac{1}{\theta+\varepsilon} \int_{0.444}^{\infty} e^{-(1-\varepsilon) t /(\theta+\varepsilon)} N^{*}(t, P) d t \leq 0.482901 . \\
& \text { 2) } 1-0.26 / \mathcal{L} \leq \beta_{\chi_{d}} \leq 1-0.24 / \mathcal{L} \Rightarrow I_{1} \leq 0.092516, I_{2} \leq 0.537213 . \\
& \text { 3) } 1-0.28 / \mathcal{L} \leq \beta_{\chi_{d}} \leq 1-0.26 / \mathcal{L} \Rightarrow I_{1} \leq 0.075429, I_{2} \leq 0.5834782 . \\
& \text { 4) } 1-0.30 / \mathcal{L} \leq \beta_{\chi_{d}} \leq 1-0.28 / \mathcal{L} \Rightarrow I_{1} \leq 0.0624122, I_{2} \leq 0.6431567 .
\end{aligned}
$$


5) $1-0.32 / \mathcal{L} \leq \beta_{\chi_{d}} \leq 1-0.30 / \mathcal{L} \Rightarrow I_{1} \leq 0.0543097, I_{2} \leq 0.714270$.

6) $1-0.34 / \mathcal{L} \leq \beta_{\chi_{d}} \leq 1-0.32 / \mathcal{L} \Rightarrow I_{1} \leq 0.0509092, I_{2} \leq 0.774367$.

7) $1-0.36 / \mathcal{L} \leq \beta_{\chi_{d}} \leq 1-0.34 / \mathcal{L} \Rightarrow I_{1} \leq 0.0520594, I_{2} \leq 0.7143177$.

8) $1-0.38 / \mathcal{L} \leq \beta_{\chi_{d}} \leq 1-0.36 / \mathcal{L} \Rightarrow I_{1} \leq 0.0581037, I_{2} \leq 0.628356$.

9) $1-0.40 / \mathcal{L} \leq \beta_{\chi_{d}} \leq 1-0.38 / \mathcal{L} \Rightarrow I_{1} \leq 0.0694366, I_{2} \leq 0.5560776$.

10) $1-0.42 / \mathcal{L} \leq \beta_{\chi_{d}} \leq 1-0.40 / \mathcal{L} \Rightarrow I_{1} \leq 0.0871545, I_{2} \leq 0.4952959$.

11) $1-0.44 / \mathcal{L} \leq \beta_{\chi_{d}} \leq 1-0.42 / \mathcal{L} \Rightarrow I_{1} \leq 0.1123271, I_{2} \leq 0.4441753$.

12) $1-0.46 / \mathcal{L} \leq \beta_{\chi_{d}} \leq 1-0.44 / \mathcal{L} \Rightarrow I_{1} \leq 0.1354119, I_{2} \leq 0.40117443$.

13) $1-0.48 / \mathcal{L} \leq \beta_{\chi_{d}} \leq 1-0.46 / \mathcal{L} \Rightarrow I_{1} \leq 0.152843, I_{2} \leq 0.364999$.

14) $1-0.50 / \mathcal{L} \leq \beta_{\chi_{d}} \leq 1-0.48 / \mathcal{L} \Rightarrow I_{1} \leq 0.164587, I_{2} \leq 0.334561$.

15) $1-0.517 / \mathcal{L} \leq \beta_{\chi_{d}} \leq 1-0.50 / \mathcal{L} \Rightarrow I_{1} \leq 0.1774831, I_{2} \leq 0.3089471$.

16) $1-0.517 / \mathcal{L} \geq \beta_{\chi_{d}} \Rightarrow I_{1} \leq 0.1774831, I_{2} \leq 0.3089471$.

Hence in all cases we have

$$
I_{1} I_{2} \leq 0.0557876 .
$$

Now we suppose that the exceptional primitive real character $\widetilde{\chi}(\bmod \widetilde{q})$ exists, and the unique exceptional real zero $\widetilde{\beta}$ of $L(s, \widetilde{\chi})$ satisfies the condition $\widetilde{\delta}(\theta+\varepsilon) \log x \leq 0.239$ where $\widetilde{\delta}=1-\widetilde{\beta}$. In this case, as above we have

$$
I_{1} \leq 0.00215731, \quad I_{2} \leq 0.39343082 .
$$

Hence we have

$$
I_{1} I_{2} \leq 0.00084876 .
$$

By the definitions of $r_{7}(n), r_{8}(n), r_{9}(n)$, just as for $D_{16}(n), D_{13}(n)$ in [1]-[3], by Cauchy's inequality we have

$$
\left|r_{7}(n)\right| \leq \sum_{r \leq P} \sum_{\chi(\bmod r)}^{*} \sum_{\substack{q \leq P \\[\tilde{q}, r] \mid q}} \frac{1}{\phi^{2}(q)}|Z(q, \tilde{\chi}, \chi)|\left\{\int_{-1 /(q Q)}^{1 /(q Q)}|\widetilde{T}(z)|^{2} d z\right\}^{1 / 2} W(\chi) .
$$

Since

$$
\int_{-1 /(q Q)}^{1 /(q Q)}|\widetilde{T}(z)|^{2} d z \leq \int_{-1}^{1}|\widetilde{T}(z)|^{2} d z \leq N \log ^{-2} N
$$

by Lemma $4,(3.8)$ and $(3.12)$ we have

$$
\begin{aligned}
\left|r_{7}(n)\right| & \leq 2.140782(1+\varepsilon) \sigma(n) N \log ^{-2} N\left(1+2 \cdot 10^{-5}\right) W(P, \widetilde{q}) \\
& \leq 0.0046185 \sigma(n) N \log ^{-2} N .
\end{aligned}
$$

Similarly

$$
\left|r_{8}(n)\right| \leq 0.0046185 \sigma(n) N \log ^{-2} N
$$


For $r_{9}(n)$, by the definition and Cauchy's inequality

$$
\left|r_{9}(n)\right| \leq \sum_{r_{1} \leq P} \sum_{\chi\left(\bmod r_{1}\right)}^{*} \sum_{r_{2} \leq P} \sum_{\chi\left(\bmod r_{2}\right)}^{*} \sum_{\substack{q \leq P \\\left[r_{1}, r_{2}\right] \mid q}} \phi(q)^{-2}|Z(q, \tilde{\chi}, \chi)| W\left(\chi_{1}\right) W\left(\chi_{2}\right) .
$$

By Lemma 4, (3.8) and (3.11)

$$
\begin{aligned}
\left|r_{9}(n)\right| & \leq 2.140782(1+\varepsilon) \sigma(n) N \log ^{-2} N\left(1+2 \cdot 10^{-5}\right) W(P) W^{\prime}(P) \\
& \leq 0.11943387 \sigma(n) N \log ^{-2} N .
\end{aligned}
$$

When $\widetilde{\beta}$ does not exist, then there is no $r_{7}(n), r_{8}(n)$. By Lemma $4,(3.8)$ and (3.13),

$$
\begin{aligned}
\left|r_{9}(n)\right| & \leq 2.140782(1+\varepsilon) \sigma(n) N \log ^{-2} N\left(1+2 \cdot 10^{-5}\right) W(P) W^{\prime}(P) \\
& \leq 0.0018171 \sigma(n) N \log ^{-2} N .
\end{aligned}
$$

By Lemma 3 and (3.3), Theorem 3 follows.

4. Proof of Theorems 1 and 2. In this section we let $L:=\log _{2} N$, and let $r_{k, k}(n)$ denote the number of representations of $n$ in the form

$$
n=2^{\nu_{1}}+\ldots+2^{\nu_{k}}-2^{\mu_{1}}-\ldots-2^{\mu_{k}}
$$

with $1 \leq \nu_{i}, \mu_{i} \leq L$.

Lemma 5. For $k \geq 2$ and $\varepsilon>0$, there exists a positive constant $N(k, \varepsilon)$ such that when $N \geq N(k, \varepsilon)$ we have

$$
\left|\sum_{m \neq 0} r_{k, k}(m) \sigma(m)-2 L^{2 k}\right| \leq 2 L^{2 k}\{H(k)+\varepsilon\},
$$

where

$$
H(k):=\min _{9 \leq E \leq L}\left\{1.7811\left(1-\frac{1}{E \csc ^{2}(\pi / 8)}\right)^{2 k} \log E+2.3270 \cdot \frac{1+\log E}{E}\right\} .
$$

This is Lemma 7 of [10].

Let

$$
G(\alpha)=\sum_{\nu \leq L} e\left(2^{\nu} \alpha\right)
$$

Lemma 6. We have

$$
\int_{0}^{1}|S(\alpha) G(\alpha)|^{2} d \alpha \leq \frac{2}{\log ^{2} 2} C N
$$

where $C<8.23382$. 
Proof. The proof is the same as that of Lemma 4 of [12]. Let

$$
s(N)=\int_{0}^{1}|S(\alpha) G(\alpha)|^{2} d \alpha .
$$

Since for fixed $l \geq 1$,

$$
\left|\left\{m_{j} \leq L:\left|m_{2}-m_{1}\right|=l\right\}\right| \leq 2(L-l),
$$

instead of (3.7) of [12] we have

$$
s(N)<2 C_{0} C_{2} \frac{N}{\log ^{2} N} \sum_{1 \leq l \leq L}(L-l) g\left(2^{l}-1\right)+\left(\frac{1}{\log 2}+\varepsilon\right) N .
$$

By the proof of Lemma 4 of [12] we have

$$
\sum_{1 \leq l \leq y} g\left(2^{l}-1\right) \leq\left(1.1160+\frac{1.4818(1+\log 10)}{10}\right) y<1.605378 y .
$$

Consequently, by Lemma 2.6 of [16],

$$
\sum_{1 \leq l \leq L}(L-l) g\left(2^{l}-1\right) \leq 0.802689 L^{2} .
$$

Hence

$$
\begin{aligned}
s(N) & \leq\left(\frac{2}{\log ^{2} 2} 0.6602 \cdot 7.8342 \cdot 1.8998 \cdot 0.802689+\frac{1}{\log 2}+\varepsilon\right) N \\
& <\frac{2}{\log ^{2} 2} 8.23382 N .
\end{aligned}
$$

The proof of Lemma 6 is complete.

Define

$$
\begin{aligned}
\Theta:=\Theta(\eta):= & \frac{1}{\log 2} \eta \csc ^{2}(\pi / 8) \log \frac{1}{\eta \csc ^{2}(\pi / 8)} \\
& +\frac{1}{\log 2}\left(1-\eta \csc ^{2}(\pi / 8)\right) \log \frac{1}{1-\eta \csc ^{2}(\pi / 8)} .
\end{aligned}
$$

Lemma 7. Let $\eta=1 / 725$. Then for $k \geq 2$ and $\varepsilon>0$, there exists a positive constant $N(k, \varepsilon)$ such that when $N \geq N(k, \varepsilon)$ we have

$$
\sum_{m \leq N}\left(r_{k}^{\prime}(m)\right)^{2} \leq \frac{2 N L^{2 k}}{\log ^{2} N}\left\{1.11943387(1+H(k))+8.23382(1-\eta)^{2 k-2}+\varepsilon\right\} .
$$

Pr o of. As in Lemma 10 of [10] (note that $\Theta(\eta)<1 / 13$ ), by Lemmas $5-7$ and Theorem 3 the lemma follows.

Proof of Theorems 1 and 2. For $k=953$, choose $E=52$. We have $H(953)<0.254146$. Note that 
$0.11943387+1.11943387 \cdot 0.254146+8.23382\left(1-\frac{1}{725}\right)^{1904}<0.9986$

Theorem 1 and Theorem 2 can now be proved in the same way as Theorem 1 and Theorem 2 in Section 7 of [11].

\section{References}

[1] J. R. Chen, The exceptional set of Goldbach numbers (II), Sci. Sinica 26 (1983), 714-731.

[2] J. R. Chen and J. M. Liu, The exceptional set of Goldbach numbers (III), Chinese Quart. J. Math. 4 (1989), 1-15.

[3] J. R. Chen and C. D. Pan, The exceptional set of Goldbach numbers, Sci. Sinica 23 (1980), 416-430.

[4] H. Z. Li, Zero-free regions for Dirichlet L-functions, Quart. J. Math. Oxford 50 (1999), 13-23.

[5] - The exceptional set of Goldbach numbers, ibid., 471-482.

[6] - , The exceptional set of Goldbach numbers (II), Acta Arith. 92 (2000), 71-88.

[7] - , The number of powers of $2 \mathrm{in}$ a representation of large even integers by sums of such powers and of two primes, ibid., 229-237.

[8] Yu. V. Linnik, Prime numbers and powers of two, Trudy Mat. Inst. Steklov. 38 (1951), 151-169 (in Russian).

[9] - Addition of prime numbers and powers of one and the same number, Mat. Sb. (N.S.) 32 (1953), 3-60 (in Russian).

[10] J. Y. Liu, M.-C. Liu and T. Z. Wang, The number of powers of 2 in a representation of large even integers (I), Sci. China Ser. A 41 (1998), 386-398.

[11] - - - - , The number of powers of 2 in a representation of large even integers (II), ibid., 1255-1271.

[12] - - - - On the almost Goldbach problem of Linnik, J. Théor. Nombres Bordeaux 11 (1999), 133-147.

[13] M.-C. Liu and K. M. Tsang, Small prime solutions of linear equations, in: Théorie des Nombres, Koninck \& Levesque (eds.), de Gruyter, 1989, 595-624.

[14] M.-C. Liu and T. Z. Wang, A numerical bound for small prime solutions of some ternary linear equations, Acta Arith. 86 (1998), 343-383.

[15] H. L. Montgomery and R. C. Vaughan, The exceptional set in Goldbach's problem, ibid. 27 (1975), 353-370.

[16] R. C. Vaughan, The Hardy-Littlewood Method, Cambridge Univ. Press, 1981.

Department of Mathematics

Shandong University

Jinan Shandong 250100

P.R. China

E-mail: lihz@sdu.edu.cn 